\title{
OPERATOR SPLITTING FOR ABSTRACT CAUCHY PROBLEMS WITH DYNAMICAL BOUNDARY CONDITIONS
}

\author{
Petra Csomós, Matthias Ehrhardt AND BÁlint FARKaS
}

Abstract. In this work we study operator splitting methods for a certain class of coupled abstract Cauchy problems, where the coupling is such that one of the sub-problems prescribes a "boundary type" extra condition for the other one. The theory of one-sided coupled operator matrices provides an excellent framework to study the well-posedness of such problems. We show that with this machinery even operator splitting methods can be treated conveniently and rather efficiently. We consider three specific examples: the Lie (sequential), the Strang, and the weighted splitting, and prove the convergence of these methods along with error bounds under fairly general assumptions. Simple numerical examples show that the obtained theoretical bounds can be computationally realised.

Mathematics subject classification (2020): 47D06, 47N40, 34G10, 65J08, 65M12, 65M15.

Keywords and phrases: Operator splitting, Lie and Strang splitting, Trotter product, abstract dynamical boundary problems, error bound.

\section{REFERENCES}

[1] R. Altmann, A PDAE formulation of parabolic problems with dynamic boundary conditions, Appl. Math. Lett. 90 (2019), 202-208.

[2] K. AtKinson And W. Han, Theoretical Numerical Analysis: A Functional Analysis Framework, Texts in Applied Mathematics, vol. 39, Springer, 2009.

[3] K. A. BAgRinovskiI AND S. K. Godunov, Difference schemes for multidimensional problems, Dokl. Akad. Nauk. USSR 115 (1957), 431-433 (Russian).

[4] A. BÁtKAi, P. Csomós, K.-J. ENGel, AND B. FARKAS, Operator splitting for operator matrices, Int. Eq. Oper. Theor. 74 (2012), 281-299.

[5] A. BÁTKAI, P. CSOMÓs, AND B. FARKAS, Operator splitting for nonautonomous delay equations, Comput. Math. Appl. 65 (2013), 315-324.

[6] A. BÁTKAI, P. CSOMÓs, AND B. FARKAS, Operator splitting for dissipative delay equations, Semigroup Forum 95 (2017), 345-365.

[7] A. BátKai, P. Csomós, B. FARKas, AND G. Nickel, Operator splitting for non-autonomous evolution equations, J. Funct. Anal. 260 (2011), 2163-2190.

[8] J. Behrndt, F. GESZTESY, AND M. MitreA, Sharp boundary trace theory and Schrödinger operators on bounded Lipschitz domains, (2020), 143 pages, preprint.

[9] M. BJøRHUS, Operator splitting for abstract Cauchy problems, IMA J. Numer. Anal. 18 (1998), 419-443.

[10] M. Caliari, A. Ostermann, and C. Piazzola, A splitting approach for the magnetic Schrödinger equation, J. Comput. Appl. Math. 316 (2017), 74-85.

[11] V. CASARino, K.-J. Engel, R. NAgel, AND G. NiCKel, A semigroup approach to boundary feedback systems, Int. Eq. Op. Th. 47 (2003), no. 3, 289-306.

[12] P. Csomós AND G. Nickel, Operator splitting for delay equations, Comput. Math. Appl. 55 (2008), 2234-2246.

[13] P. Csomós, A. BÁtKai, B. Farkas, And A. Ostermann, Operator semigroups fur numerical analysis, Lecture notes, TULKA Internetseminar,

https://www.math.tecnico.ulisboa.pt/ czaja/ISEM/15internetseminar201112.pdf, 2012, p. 182 pages. 
[14] P. CSOMós, I. FARAGó, AND A. HAVASI, Weighted sequential splitting and their analysis, Comput. Math. Appl. 50 (2005), no. 7, 1017-1031.

[15] I. Dimov, I. Faragó, A. Havasi, AND Z. Zlatev, Different splitting techniques with application to air pollution models, Int. J. Environment. Pollution 32 (2008), 174-199.

[16] J. EILINGHOFF AND R. SCHNAUBELT, Error analysis of an ADI splitting scheme for the inhomogeneous Maxwell equations, Discrete Contin. Dyn. Syst. 38 (2018), no. 11, 5685-5709.

[17] K.-J. ENGEL, Matrix representation of linear operators on product spaces, no. 56, 1998, International Workshop on Operator Theory (Cefalù, 1997), pp. 219-224.

[18] K.-J. ENGEL, Spectral theory and generator property for one-sided coupled operator matrices, Semigroup Forum 58 (1999), no. 2, 267-295.

[19] K.-J. ENGEL AND R. NAGEL, One-parameter semigroups for linear evolution equations, Graduate Texts in Mathematics, vol. 194, Springer-Verlag, New York, 2000.

[20] Y. EPSHTEYN AND Q. XIA, Difference potentials method for models with dynamic boundary conditions and bulk-surface problems, Adv. Comput. Math. 46 (2020).

[21] T. FukaO, S. YoshikaWa, AND S. Wada, Structure-preserving finite difference schemes for the Cahn-Hilliard equation with dynamic boundary conditions in the one-dimensional case, Commun. Pure Appl. Anal. 16 (2017), 1915-1938.

[22] J. GEISER, Iterative Splitting Methods for Differential Equations, Chapman and Hall/CRC Numerical Anal. and Sci. Comp. Series, CRC Press, Hoboken, NJ, 2011.

[23] F. Gesztesy, I. Mitrea, D. Mitrea, And M. Mitrea, On the nature of the Laplace-Beltrami operator on Lipschitz manifolds, vol. 172, 2011, Problems in mathematical analysis, no. 52, pp. 279346.

[24] F. Gesztesy and M. Mitrea, Generalized Robin boundary conditions, Robin-to-Dirichlet maps, and Krein-type resolvent formulas for Schrödinger operators on bounded Lipschitz domains, Perspectives in partial differential equations, harmonic analysis and applications, Proc. Sympos. Pure Math., vol. 79, Amer. Math. Soc., Providence, RI, 2008, pp. 105-173.

[25] F. Gesztesy AND M. Mitrea, A description of all self-adjoint extensions of the Laplacian and Krĕn-type resolvent formulas on non-smooth domains, J. Anal. Math. 113 (2011), 53-172.

[26] G. GreInER, Perturbing the boundary conditions of a generator, Houston J. Math. 13 (1987), no. 2, 213-229.

[27] M. HAASE, The functional calculus for sectorial operators, Operator Theory: Advances and Applications, vol. 169, Birkhäuser Verlag, Basel, 2006.

[28] E. Hansen And A. Ostermann, Dimension splitting for evolution equations, Numer. Math. 108 (2008), 557-570.

[29] E. HANSEN AND A. OSTERMANn, High order splitting methods for analytic semigroups exist, BIT 49 (2009), no. 3, 527-542.

[30] E. Hansen AND A. Ostermann, Dimension splitting for quasilinear parabolic equations, IMA J. Numer. Anal. 30 (2010), no. 3, 857-869.

[31] D. HipP, A unified error analysis for spatial discretizations of wave-type equations with applications to dynamic boundary conditions, Ph. D. thesis, Karlsruher Institut für Technologie (KIT), 2017.

[32] D. Hipp AND B. KovÁCs, Finite element error analysis of wave equations with dynamic boundary conditions: $L^{2}$ estimates, IMA J. Numer. Anal. 41 (2020), no. 1, 638-728.

[33] M. Hochbruck, T. JAhnKe, AND R. Schnaubelt, Convergence of an ADI splitting for Maxwell's equations, Numer. Math. 129 (2015), no. 3, 535-561.

[34] H. Holden, C. Lubich, AND N. H. Risebro, Operator splitting for partial differential equations with Burgers nonlinearity, Math. Comp. 82 (2013), 173-185.

[35] W. Hundsdorfer And J. G. Verwer, Solution of Time-dependent Advection-Diffusion-Reaction Equations, Springer Series in Computational Mathematics, vol. 33, Springer, 2003.

[36] T. JAHNKE AND C. LUBICH, Error bounds for exponential operator splittings, BIT 40 (2000), no. 4, $735-744$.

[37] T. JAhnke, M. MikL, AND R. Schnaubelt, Strang splitting for a semilinear Schrödinger equation with damping and forcing, J. Math. Anal. Appl. 455 (2017), no. 2, 1051-1071.

[38] E. R. Jakobsen, K. Hvistendahl Karlsen, And N. H. Risebro, On the convergence rate of operator splitting for Hamilton-Jacobi equations with source terms, SIAM J. Numer. Anal. 39 (2001), no. 2, 499-518. 
[39] P. KNOPF AND K. F. LAM, Convergence of a Robin boundary approximation for a Cahn-Hilliard system with dynamic boundary conditions, Nonlinearity 33 (2020), no. 8, 4191-4235.

[40] P. KNOpF, K. F. LAm, C. LiU, And S. MetzGer, Phase-field dynamics with transfer of materials: The Cahn-Hillard equation with reaction rate dependent dynamic boundary conditions, ESAIM: M2AN 55 (2021), no. 1, 229-282.

[41] P. KNOPF AND A. Signori, On the nonlocal Cahn-Hilliard equation with nonlocal dynamic boundary condition and boundary penalization, J. Diff. Eq. 280 (2021), 236-291.

[42] B. KovÁcs, B. Li, AND C. LUBICH, Convergence of finite elements on an evolving surface driven by diffusion on the surface, Numer. Math. 137 (2017), 643-689.

[43] B. KovÁCs AND C. LUBICH, Numerical analysis of parabolic problems with dynamic boundary conditions, IMA J. Numer. Anal. 37 (2017), 1-39.

[44] F. LANGA AND M. PIERRE, A doubly splitting scheme for the caginalp system with singular potential and dynamic boundary conditions, HAL preprint hal-02310210 (2019).

[45] P. D. LAX AND R. D. RICHTMYER, Survey of the stability of linear finite difference equations, CPAM 9 (1956), 267-293.

[46] J.-L. LiONS AND E. MAGENES, Non-homogeneous boundary value problems and applications, Vol. I, Springer-Verlag, New York-Heidelberg, 1972, Translated from the French by P. Kenneth, Die Grundlehren der mathematischen Wissenschaften, Band 181.

[47] A. LUNARDI, Analytic semigroups and optimal regularity in parabolic problems, Modern Birkhäuser Classics, Birkhäuser/Springer Basel AG, Basel, 1995, (2013 reprint of the 1995 original).

[48] G. I. MARCHUK, Some application of splitting-up methods to the solution of mathematical physics problems, Applik. Mat. 13 (1968), no. 2, 103-132.

[49] W. MCLEAN, Strongly elliptic systems and boundary integral equations, Cambridge University Press, Cambridge, 2000.

[50] D. Mugnolo, A note on abstract initial boundary value problems, Tübinger Berichte zur Funktionalanalysis 10 (2001), 158-162.

[51] D. Mugnolo, Second order abstract initial-boundary value problems, Ph. D. thesis, Universität Tübingen, 2004.

[52] B. SpORTISSE, An analysis of operator splitting techniques in the stiff case, J. Comput. Phys. 161 (2000), 140-168.

[53] G. Strang, On the construction and comparison of difference schemes, SIAM J. Numer. Anal. 5 (1968), no. 3, 506-517.

[54] H. F. Trotter, On the product of semi-groups of operators, Proc. Amer. Math. Soc. 10 (1959), $545-551$. 